\title{
Role of Annexin A2 in the EGF-induced epithelial-mesenchymal transition in human CaSki cells
}

\author{
LEI CUI $^{1 *}$, JIAN SONG ${ }^{2 *}$, LITING WU ${ }^{3}$, LUHUI CHENG ${ }^{1}$, AIJUN CHEN ${ }^{1}$, \\ YANLIN WANG ${ }^{3}$, YINGDI HUANG ${ }^{3}$ and LIMING HUANG ${ }^{3}$
}

\begin{abstract}
${ }^{1}$ Department of General Surgery, Yichang Central People's Hospital, The First College of Clinical Medical Science, Three Gorges University, Yichang, Hubei 443002; ${ }^{2}$ Department of Biochemistry, School of Basic Medical Sciences, Wuhan University, Wuhan, Hubei 430071; ${ }^{3}$ Institute of Molecular Biology of Three Gorges University, Yichang, Hubei 443002, P.R. China
\end{abstract}

Received June 11, 2015; Accepted October 18, 2016

DOI: $10.3892 / \mathrm{ol} .2016 .5406$

\begin{abstract}
The epidermal growth factor receptor (EGF-R) signaling pathway is thought to have an important role in the development and progression of several carcinomas, as it is associated with cell proliferation, differentiation and migration. Activation of EGF-R signaling regulates epithelial-mesenchymal transition (EMT)-associated invasion and migration in normal and malignant epithelial cells. However, the specific mechanisms have not yet been fully elucidated. The present study utilized wound healing assays, western blotting, flow cytometry and MTT assays to demonstrate that Annexin A2 (ANXA2) is a key regulatory factor in EGF-induced EMT in CaSki cervical cancer cells. Moreover, the increased expression levels of ANXA2 promoted cell viability and migration in human CaSki cells. It was also found that silencing ANXA2 partially reverses EGF-induced EMT and inhibits cell viability and migration in CaSki cells. These findings suggest that ANXA2 is a key regulator of EGF-induced EMT in CaSki cervical cancer cells.
\end{abstract}

\section{Introduction}

Cervical cancer is the second most common type of cancer among women worldwide, with a mortality rate of $\sim 300,000$ annually (1). Metastasis is responsible for the vast burden of

Correspondence to: Dr Lei Cui, Department of General Surgery, Yichang Central People's Hospital, The First College of Clinical Medical Science, Three Gorges University, Yichang, Hubei 443002, P.R. China

E-mail: leileicui@126.com

Professor Liming Huang, Institute of Molecular Biology of Three Gorges University, Yichang, Hubei 443002, P.R. China

E-mail: hlmyj8265@sina.com

${ }^{*}$ Contributed equally

Key words: Annexin A2, epithelial-mesenchymal transition, epidermal growth factor receptor signaling, cell proliferation cancer-associated morbidity and mortality. Overexpression of epithelial growth factor receptor (EGF-R) is detected in $70-90 \%$ of all cervical cancer cases (2-4). EGF-R activation induces the epithelial-mesenchymal transition (EMT), which is accompanied by the overexpression of mesenchymal markers. In contrast, EGF-R inhibition, by a tyrosine kinase inhibitor or antibody, provokes EMT, which is accompanied by the upregulation of epithelial-marker proteins, such as E-cadherin (E-cad) and Zonula occludens-1 (5). The EMT is a multi-step process that includes dysfunctional cell-cell adhesive interactions, loss of cell-cell junctions and reorganization of the cytoskeleton, which is associated with cell proliferation, metastasis and immune escape.

Annexin A2 (ANXA2), a calcium-dependent phospholipid binding protein, is abundantly present in various cancer cells, and has multiple roles in regulating cellular function, particularly tumor differentiation, clinical outcomes and metastatic potential $(6,7)$. Previous studies have implicated ANXA2 in various biological functions, including mitogenic signal transduction, fibrinolysis, immune response, proliferation, carcinogenesis and tumor progression (7-11). ANXA2 has been demonstrated to be a co-receptor for both plasminogen and tissue-type plasminogen activator, which cleaves inactive plasminogen to yield the active serine proteinase, plasmin $(12,13)$. Subsequent studies elucidated that the conversion of plasminogen to plasmin is induced by ANXA2 promoting metastasis, which leads to the activation of metalloproteinases, degradation of extracellular matrix components, and promotion of neoangiogenesis (14-17). However, the underlying mechanisms of ANXA2 and EMT remain obscure.

The present study utilized wound healing assays, western blotting, flow cytometry and MTT assays to demonstrate that ANXA2 is a key regulatory factor of EGF-induced EMT in CaSki cervical cancer cells.

\section{Materials and methods}

Cell lines. CaSki, HeLa and $\mathrm{SiHa}$ cells were purchased from the Cell Bank of China (Wuhan, China) and were cultured under standard conditions in RPMI-1640 medium (Shanghai 
Biosun Sci\&Tech Co., Ltd., Shanghai, China) supplemented with 10\% fetal bovine serum (Gibco; Thermo Fisher Scientific, Inc., Waltham, MA, USA), $100 \mu \mathrm{g} / \mathrm{ml}$ streptomycin and 100 units $/ \mathrm{ml}$ penicillin. The cell lines were maintained in a humidified incubator containing $5 \% \mathrm{CO}_{2}$ at $37^{\circ} \mathrm{C}$. The cells were passaged twice a week at an initial density of $1 \times 10^{6}$ cells $/ \mathrm{ml}$. CasKi cells were cultured with various concentrations $(0,5,10,50$ and $100 \mathrm{ng} / \mathrm{ml}$ ) of EGF (Santa Cruz Biotechnology Inc., Dallas, TX, USA).

Plasmid construction and stableltransient transfection. The human genomic fragment of ANXA2, amplified by nested-polymerase chain reaction (PCR) and $\sim 1,076 \mathrm{bp}$, was cloned into the pcDNA3.1 (+) vector. Primers for nested-PCR of ANXA2 were as follows: Sense 5'-CAGCATTTGGGG ACGCTCTCAGC-3' and anti-sense 5'-ATTTCTGGACGC TCAGGCCGTGT-3'; sense 5'-TCC TCGAGCATTTGGGGA CGCTCTCAGCTCTC-3' and anti-sense 5'-GCGGATCCC TTCAGTCATCTCCACCACACAGG-3'. To generate a cell line that stably expresses ANXA2, CaSki cells were transfected with pcDNA3.1-ANXA2 using Lipofectamine 2000 reagent (Invitrogen; Thermo Fisher Scientific, Inc.). Following selection with G418, a single clone that overexpressed ANXA2 was identified by western blotting. To knockdown ANXA2, the ANXA2 siRNA reagent (sc-270151; Santa Cruz Biotechnology Inc.) was used for transfection.

Wound healing assay. Cells were plated at $2 \times 10^{5} / 1$ cells per well in a 6-well plate and grown overnight under standard conditions. A straight-line scratch was made on a confluent monolayer of cells using a sterile 1-ml disposable serological pipette. To remove debris and to smooth the edge of the scratch, the cells were washed with $1 \mathrm{ml}$ PBS. Images of cell proliferation were captured using a Nikon Eclipse TS100 microscope (Nikon Corp., Tokyo, Japan) at 0, 24 and $48 \mathrm{~h}$ after the scratch was made.

Western blot. Total protein was extracted from cells using cell lysis buffer (0.5\% NP-40, 0.5\% SDS, 1.5 Mm Tris-HC, pH 7.4 and $15 \mathrm{mM} \mathrm{NaCl})$. Protein samples $(20 \mu \mathrm{g} / \mathrm{lane})$ were separated by $5 \%$ (spacer gel) and then 10\% (separation gel) SDS-PAGE, and transferred to polyvinylidene difluoride membranes. Following blocking with 5\% skimmed milk, the membranes were incubated overnight with primary antibodies against E-cadherin (sc-7870; 1:1,000; Santa Cruz Biotechnology, Inc.), N-cad (BA0637; 1:500; Boster Biological Technology, Ltd., Wuhan, China), ANXA2 (A2485; 1:3,000; Sigma-Aldrich; Merck Millipore, Darmstadt, Germany) and $\beta$-actin (sc-7210; Santa Cruz Biotechnology, Inc.). The membranes were treated with goat-anti rabbit horseradish peroxidase-conjugated secondary antibody (A32732; 1:1,000; Invitrogen; Thermo Fisher Scientific, Inc.) for $1 \mathrm{~h}$ at $37^{\circ} \mathrm{C}$. Target protein bands were determined using the enhanced chemiluminescence (ECL) reagents provided in the ECL+PLUS kit (GE Healthcare Bio-Sciences, Pittsburgh, PA, USA). Protein bands were quantified using Quantity One 4.62 software (Bio-Rad Laboratories, Inc., Hercules, CA, USA). $\beta$-actin was used as an internal control.

Flow cytometry. Flow cytometric analysis was used to determine the distribution of cells in the cell cycle sub-phases. Cells were harvested in the logarithmic growth phase and fixed overnight with $80 \%$ ethanol. Cells were washed with cold PBS and stained with propidium iodide $(0.05 \mathrm{mg} / \mathrm{ml})$ and RNase A $(0.5 \mathrm{mg} / \mathrm{ml})$ and were analyzed using a flow cytometer.

MTT assay. Cells with different ANXA2 expression levels were seeded at a density of $1 \times 10^{5}$ cells into 96 -well culture plates and grown to $40 \%$ confluence. The medium in each well was removed, and $20 \mu \mathrm{l}$ MTT solution $(5 \mathrm{mg} / \mathrm{ml})$ was added to each well. Following incubation at $37^{\circ} \mathrm{C}$ for $4 \mathrm{~h}$, the medium was removed and $150 \mu 1 \mathrm{DMSO}$ was added to each well. The optical density at $570 \mathrm{~nm}$ was recorded. The cell growth rate was calculated as follows:

Cell growth rate $=$ ANh $/$ A0h $\times 100 \%(\mathrm{~N} \sim 24,48,72,96$, 120).

Statistical analysis. All data are presented as the mean \pm standard deviation. Statistical analysis between the groups was assessed using Student's two-tailed t-test and analysis of variance. $\mathrm{P}<0.05$ was considered to indicate a statistically significant difference.

\section{Results}

EGF induces EMT and promotes cell viability. Different tumor cell lines exhibit different metastasis properties, which are associated with the expression levels of E-cad (18). Initially, E-cad expression levels were tested in different cervical cancer cell lines, including $\mathrm{HeLa}, \mathrm{SiHa}$ and $\mathrm{CaSki}$. E-cad expression levels in CaSki cells were markedly increased, as compared with SiHa and HeLa cells (Fig. 1A). Due to this, CaSki cells were used for the epithelial model and were cultured under standard conditions with or without EGF $(100 \mathrm{ng} / \mathrm{ml})$ for $24 \mathrm{~h}$. Morphological changes were assessed. After $48 \mathrm{~h}$ of EGF $(100 \mathrm{ng} / \mathrm{ml})$ treatment, CaSki cells became more fusiform and the connections between the cells decreased (Fig. 1B). Moreover, the E-cad and N-cad expression levels were analyzed by western blotting, which suggesed that EGF treatment was able to upregulate N-cad expression levels and downregulate E-cad expression levels (Fig. 1C). EGF treatment disrupted the cell cycle distribution by decreasing the G0/G1 phase (normal, $70.9 \%$; EGF treatment, 56.9\%) and increasing the G2/M (normal, 7.6\%; EGF treatment, 13.3\%) and S phases (normal, 21.5\%; EGF treatment, 29.7\%) under EGF treatment (Fig. 1D). These data indicate that EGF may induce EMT and promote cell viability by interfering with the cell cycle. Similar results have been published previously (19).

EGF treatment upregulates ANXA2. Previous studies have illustrated that ANXA2 is involved in the EMT process (20). To investigate the association between ANXA2 and EGF treatment, total protein was collected from different CaSki cells that were treated with different EGF concentrations ( 0 , 5, 10, 50 and $100 \mathrm{ng} / \mathrm{ml}$; Fig. 2A) or with different treatment durations (0, 12, 24, 36 and 48 h; Fig. 2B). Expression levels of ANXA2 after $24 \mathrm{~h}$ of $100 \mathrm{ng} / \mathrm{ml}$ EGF treatment were significantly higher than that of cells without EGF treatment or after $24 \mathrm{~h}$ at a lower concentration $(\mathrm{P}<0.01)$. Furthermore, 
A

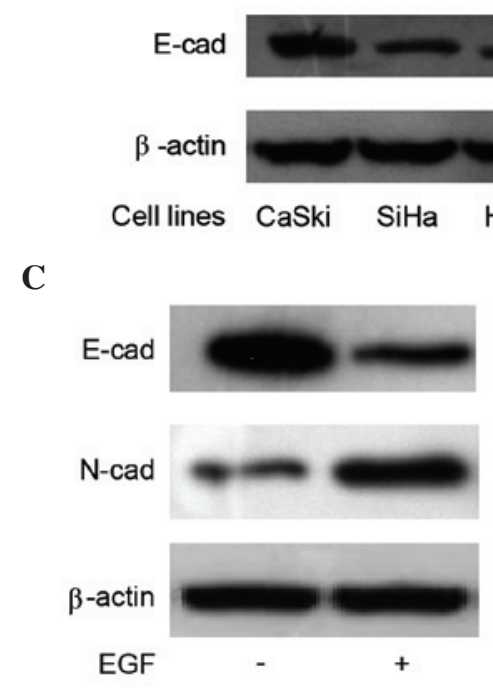

B

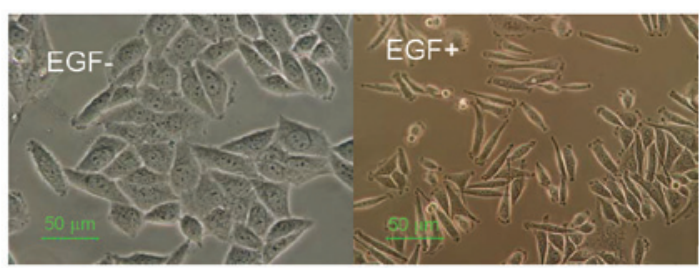

D

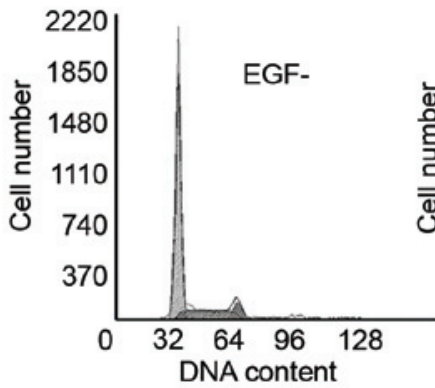

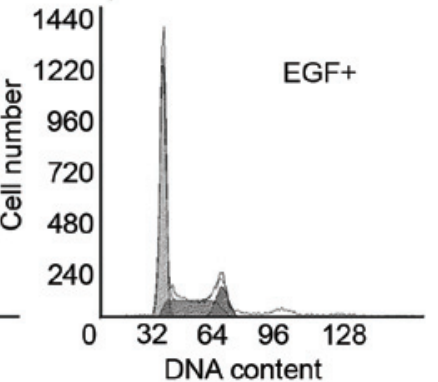

Figure 1. EGF induces EMT and promotes cell proliferation. (A) E-cad expression levels were determined by western blot analysis, and the expression levels in CaSki were much higher than that of SiHa and HeLa cells. (B) Morphological changes were detected following $24 \mathrm{~h}$ EGF (100 ng/ml) treatment. CaSki cells became more fusiform after EGF treatment. (C) E-cad and N-cad expression levels were tested by western blot analysis. EGF treatment induced downregulation of E-cad and upregulation of N-cad in CaSki cells. (D) Cell cycle distribution was tested by flow cytometry. Cell cycle distribution was disrupted by decreasing the G0/G1 phase (normal, 70.9\%; EGF, 56.9\%) and increasing the G2/M (normal, 7.6\%; EGF, 13.3\%) and S phases (normal, 21.5\%; EGF, 29.7\%) with EGF treatment. EGF, epidermal growth factor; EMT, epithelial-mesenchymal transition; E-cad, E-cadherin; N-cad, N-cadherin.

ANXA2 expression levels were higher when treated with EGF $(100 \mathrm{ng} / \mathrm{ml})$ for a longer duration. These results suggested that EGF promotes ANXA2 expression, and that EGF expression levels in cells are increased by prolonged exposure or exposure to an increased concentration (Fig. 2).

Roles of ANXA2 in EMT, growth and migration. Although ANXA2 was significantly upregulated in the EGF-induced EMT process, it is possible that this is an unrelated phenomenon or a compensatory event. To clarify the role of ANXA2 in the EMT process, CaSki cell lines that had ANXA2 overexpression or knockdown were established by transfecting pcDNA 3.1-ANXA2 or ANXA2 siRNAs, which was evaluated by western blot analysis (Fig. 3A). Using these cell lines as a model, it was demonstrated that ANXA2 downregulated E-cad expression, which was similar to the effect noted after $24 \mathrm{~h}$ of EGF $(100 \mathrm{ng} / \mathrm{ml})$ treatment. The downregulation of E-cad induced by EGF was partially reversed by ANXA2 siRNA transfection. N-cad, which is a mesenchymal marker protein and is upregulated with EGF treatment, was upregulated by ANXA2 overexpression. Furthermore, the upregulation of $\mathrm{N}$-cad induced by EGF was partially reversed by ANXA2 siRNA transfection (Fig. 3B). With overexpression of ANXA2, the cell morphology became more fusiform, and the morphological changes induced by 24-h treatment with EGF $(50 \mathrm{ng} / \mathrm{ml})$ were partially reversed by ANXA2 siRNA transfection (Fig. 3C).

Moreover, ANXA2 promoted cell growth and interfered with the cell cycle by decreasing the cell ratio in $\mathrm{G} 0 / \mathrm{G} 1$ phase (normal, 70.9\%; ANXA2 upregulation, 53.7\%), but it increased the cell ratio in S phase (normal, 21.5\%; ANXA2 upregulation, 29.6\%) and G2/M phase (normal, 7.6\%; ANXA2 upregulation, $16.8 \%$ ), which was similar to EGF treatment. However, knockdown of ANXA2 had the opposite effect compared with normal CaSki cells, which involved an increased cell ratio in the G0/G1 phase (ANXA2 downregulation, 79.1\%) and a decreased cell ratio in S phase (ANXA2 downregulation, 11.1\%) and G2/M phase (ANXA2 downregulation, 9.8\%; Figs. 3D and 4).

The results of the present study suggested that overexpression of ANXA2 may promote EMT, which was upregulated in the EGF-induced EMT process (Fig. 3). To further investigate the role of ANXA2 in migration, a wound healing assay was evaluated in normal, EGF-treated, ANXA2-upregulated and ANXA2-downregulated CaSki cell lines. These results suggest that upregulated ANXA2 or EGF may promote CaSki cell migration compared with normal cells, but silencing of ANXA2 had the opposite effect (Fig. 5).

\section{Discussion}

EMT is a dynamic process that can be regulated and reversed by various factors, including miRNA (21). Alterations in cell morphology and function during the EMT process are accompanied by changes in protein expression profiles, including the loss of epithelial markers and the de novo expression of mesenchymal markers. Previous studies have revealed that EMT can be triggered by the interplay of extracellular signals, including extracellular matrix components and soluble growth factors, such as transforming growth factor- $\beta$ and fibroblast growth factor families, EGF, insulin-like growth factor and scatter factor/hepatocyte growth factor in cancer progression $(22,23)$.

The present study explored the possible mechanism of EGF-induced EMT, in addition to how ANXA2 has a crucial role in CaSki progression. The overexpression of EGF-R is an independent predictor for poor prognosis in cervical cancer (24). Moreover, EGF-R overexpression is associated with a poor 
A

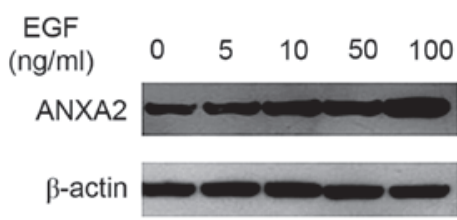

B

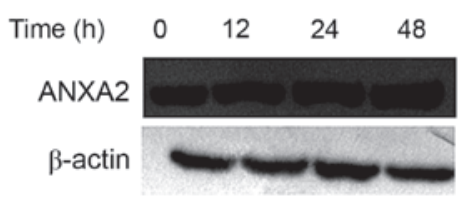

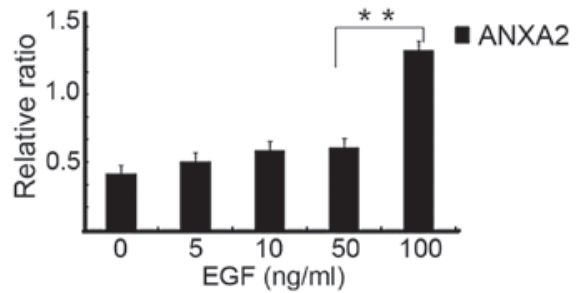

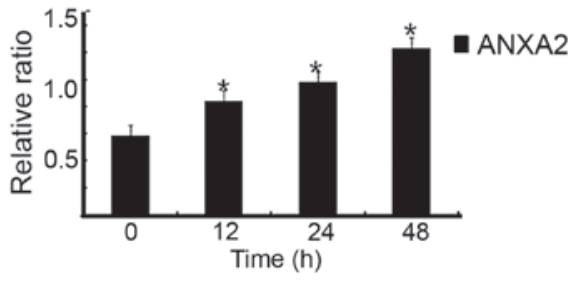

Figure 2. EGF treatment upregulates ANXA2. ANXA2 expression levels were analyzed by western blot analysis. CaSki cells were cultured with (A) various EGF concentrations $(0,5,10,50$, and $100 \mathrm{ng} / \mathrm{ml})$ for $24 \mathrm{~h}$ or (B) with EGF $(100 \mathrm{ng} / \mathrm{ml})$ treatment for different time periods $(0-48 \mathrm{~h})$. Expression levels of ANXA2 after $24 \mathrm{~h}$ of $100 \mathrm{ng} / \mathrm{ml}$ EGF treatment were higher than without EGF treatment or with a lower concentration for $24 \mathrm{~h}$. ANXA2 expression levels were increased after a longer duration of EGF $(100 \mathrm{ng} / \mathrm{ml})$ treatment. Densitometric analysis of three independent western blots. Values are presented as the means of three trials with the standard deviation indicated by error bars. " $\mathrm{P}<0.05$ and ${ }^{* *} \mathrm{P}<0.01$ vs. the control. EGF, epidermal growth factor; EMT, epithelial-mesenchymal transition; E-cad, E-cadherin; N-cad, N-cadherin; ANXA2, Annexin A2.

A
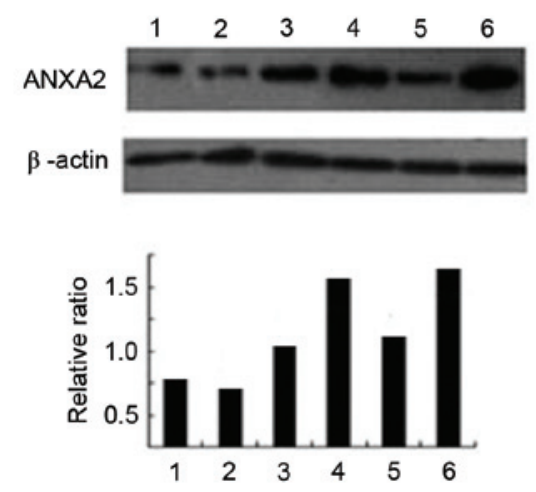

C

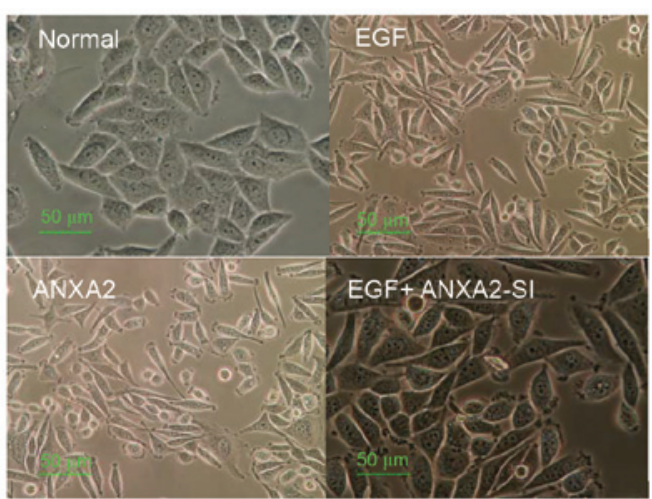

B

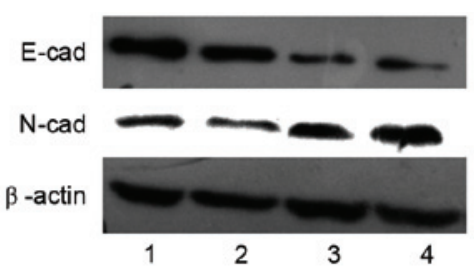

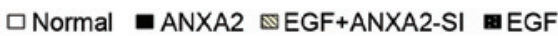

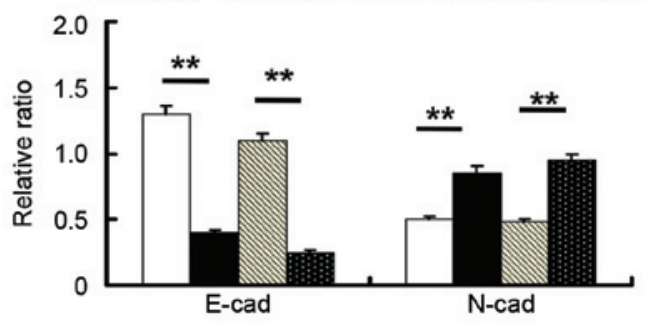

D

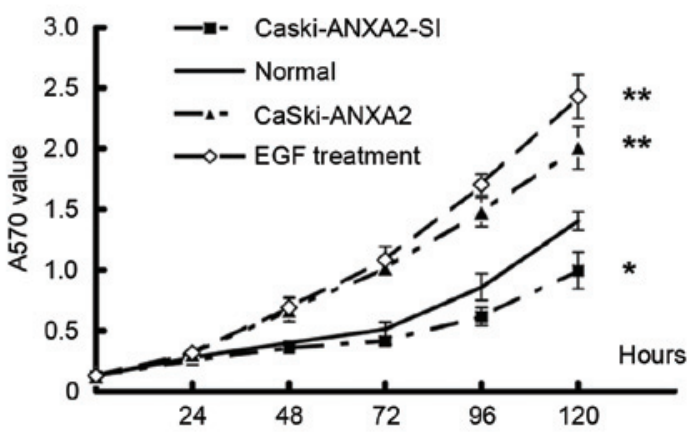

Figure 3. Roles of ANXA2 in the EMT process. To clarify the role of ANXA2 in the EMT process, CaSki cell lines with overexpressed or knocked down ANXA2 were created by transfecting pcDNA 3.1-ANXA2 or ANXA2 siRNAs. (A) Overexpression or knockdown of ANXA2 was verified by western blot analysis. 1 and 2 were transfected with ANXA2 siRNA, 3 was normal CaSki cells, 5 was transfected with pcDNA 3.1, and 4 and 6 were transfected with pcDNA3.1-ANXA2. (B) E-cad and N-cad expression levels were tested by western blot analysis; 1 was normal CaSki cells, 2 was knockdown of ANXA2 in CaSki cells with $24 \mathrm{~h}$ of EGF $(100 \mathrm{ng} / \mathrm{ml})$ treatment, 3 was forced expression of ANXA2 in CaSki cells, 4 was normal CaSki cells with EGF treatment. Forced ANXA2 expression downregulated E-cad expression levels and upregulated $\mathrm{N}$-cad expression levels, which was similar to $24 \mathrm{~h}$ of EGF (100 ng/ml) treatment. However, the upregulation of $\mathrm{N}$-cad and downregulation of E-cad induced by EGF was partially reversed by knockdown of ANXA2. Densitometric analysis of three independent western blots. Values are presented as the means of three trials with the standard deviation indicated by error bars. ${ }^{* *} \mathrm{P}<0.01$ vs. the control. (C) Morphological changes with ANXA2 transfection. Morphology was more fusiform after ANXA2 overexpression, and the morphological changes induced by $24 \mathrm{~h}$ of EGF (50 ng/ml) treatment were partially reversed by ANXA2 siRNA transfection. (D) Cell viability was analyzed by MTT assay, which showed that forced ANXA2 expression promoted cell growth, which was similar to EGF treatment. $\mathrm{P}<0.01$ vs. the normal cells. However, knockdown of ANXA2 inhibited cell growth. P<0.05 vs. the normal cells. EGF, epidermal growth factor; EMT, epithelial-mesenchymal transition; E-cad, E-cadherin; N-cad, N-cadherin; ANXA2, Annexin A2. 

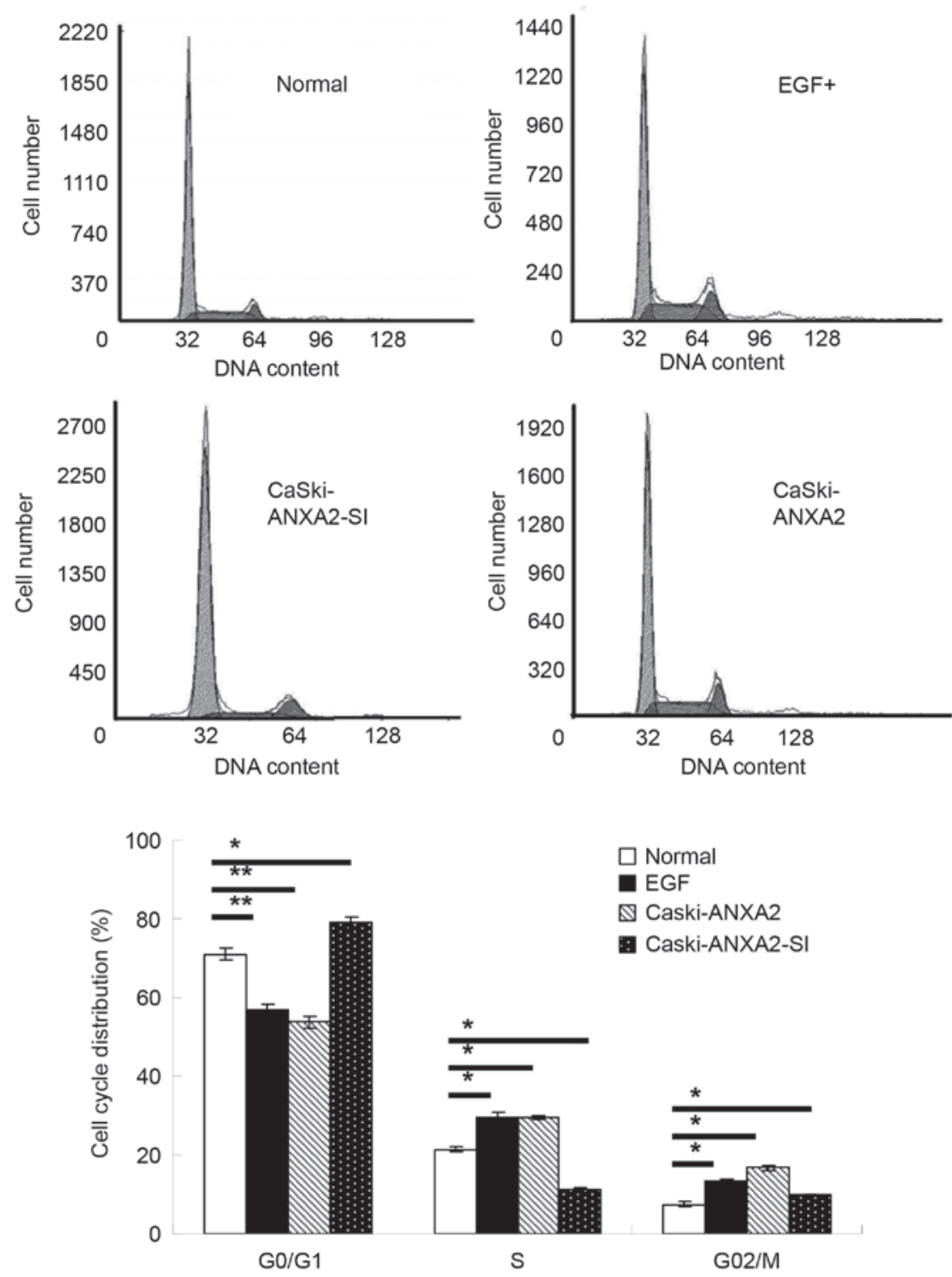

There was a upregulation, 29.6\%) and G2/M phase (norma, 7.6\%; ANXA2 upregulation, 16.8\%) when ANXA2 expression was forced. With ANXA2 knockdown, the cell ratio in G0/G1 phase increased (ANXA2 upregulation, 79.1\%), and decreased in S phase (ANXA2 upregulation, 11.1\%) and G2/M phase (ANXA2 downregulation, 9.8\%), which was evaluated using flow cytometry. Treatment with EGF (100 ng/ml) for $24 \mathrm{~h}$ was used as a positive control, and normal CaSki cells were used as a normal control. Values are presented as the means of three trials with the standard deviation indicated by error bars. ${ }^{*} \mathrm{P}<0.05$ and ${ }^{* * *} \mathrm{P}<0.01$ vs. the control cells. EGF, epidermal growth factor; EMT, epithelial-mesenchymal transition; ANXA2, Annexin A2.

response to chemoradiation (25). Initially, it was suggested that the CaSki cell line has higher E-cad expression levels than other cervical cancer cell lines, such as $\mathrm{SiHa}$ and HeLa cells. As a result, CaSki cells may be epithelial-like cells in cervical cancer, and thus were used as a model cell line in the present study. The present results demonstrated that EGF-induced EMT is accompanied by high levels of ANXA2 expression. With EGF treatment, these cells became more spindle-shaped and had mesenchymal-associated molecular profiles, such as de novo expression of $\mathrm{N}$-cadherin and increased migration activity. Grewal and Enrich (26) suggested that ANXA2 is downstream of the EGF-R signal pathway. To clarify the regulatory role of ANXA2 in EGF treatment, the role of ANXA2 in the viability and migration of human CaSki cervical cancer cells was investigated. Stable expression of ANXA2 significantly promotes cell growth by interfering with the cell cycle in vitro. However, silencing of ANXA2 inhibited the cell growth and distribution of the $\mathrm{S}$ phase less than in normal cells. Overexpression of ANXA2 was able to promote cell migration activity, whereas depletion of ANXA2 inhibited migration. It has also been suggested that ANXA2 maintains constitutive activation of EGF-R downstream signaling intermediates, contributing to cell proliferation, migration and viability (27), which is considered a potential factor that regulates cell growth, invasion and chemo-resistance (20). ANXA2 may facilitate cell proliferation by regulating p53 via c-Jun N-terminal kinase/c-Jun in HCC since disruption of the p53/miRNA-34 axis causes abnormal apoptosis and progression (28).

The present results also indicated that the overexpression of ANXA2 induces E-cad downregulation and N-cad 

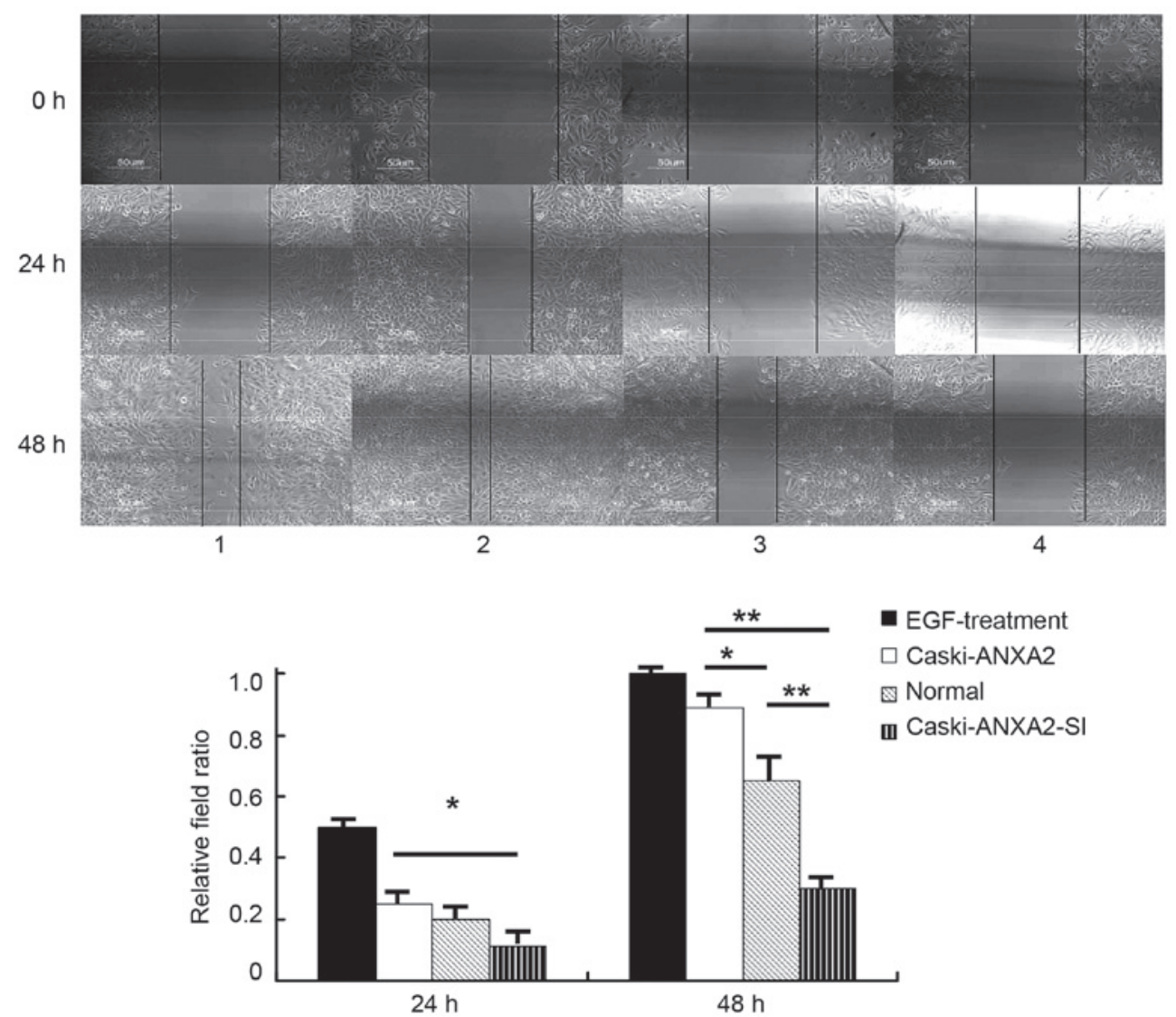

Figure 5. Wound healing assay. CaSki cells that were treated with EGF or overexpression or knockdown of ANXA2 were plated at 2x105/1 cells per well in a 6-well plate and cultured overnight under standard conditions. A straight-line scratch was made on a confluent monolayer of cells using a sterile, 1-ml, disposable serological pipette. Images of cell proliferation were captured and the close field was calculated using a Nikon Eclipse TS100 microscope at 0, 24 and $48 \mathrm{~h}$ after the scratch. Sample 1 was normal CaSki cells, 2 was CaSki cells with EGF treatment, 3 was CaSki cell with ANXA2 overexpression and 4 was CaSki cells with downregulated ANXA2. The relative close field ratio of ANXA2 overexpressed cells was much higher than that of the normal and ANXA2 knockdown cells at $48 \mathrm{~h}$ after scratching. EGF treatment was used as a positive control. Values are presented as the means of three trials with the standard deviation indicated by error bars. "P<0.05 and ${ }^{* *} \mathrm{P}<0.01$. EGF, epidermal growth factor; EMT, epithelial-mesenchymal transition; E-cad, E-cadherin; N-cad, $\mathrm{N}$-cadherin; ANXA2, Annexin A2.

upregulation with structural alterations, which is similar to EGF treatment alone. However, silencing ANXA2 reversed the downregulation of E-cad and upregulation of $\mathrm{N}$-cad that was induced by EGF treatment. In mesenchymal-like cells, downregulation of E-cadherin or upregulation of N-cad is characterized as the major hallmark responsible for the loss of cell-cell contacts in EMT events (21). E-cadherin, which is present in mature adherens junctions, is a pivotal molecule that maintains epithelial cell polarity. E-cadherin binds to $\beta$-catenin and forms a protein complex that links to the actin cytoskeleton. E-cadherin has anti-proliferation, anti-invasion and anti-metastasis functions, and loss of E-cadherin contributes to metastatic dissemination in numerous cancer types (5). The mechanisms of E-cadherin loss in malignant cancers include genetic mutation, epigenetic silencing, transcription repression and proteolytic processes (4). Another research group has also demonstrated that upregulation of ANXA2 is accompanied by the EMT process in endometrial cells, and forced expression of ANXA2 may mediate phenotypic mesenchymal-like cellular changes with structural and functional alteration in a $\beta$-catenin/TCF signal-associated manner (29). This could be reversed by inhibition of ANXA2 expression, and another study suggested that ANXA2 is closely associated with tumor progression in HeLa cells (30).
It has previously been suggested that ANXA2 depletion delays EGF-R endocytic trafficking via cofilin activation and enhances EGF-R signaling and metastasis formation (27). However, this data also suggested that this inhibition coincides with enhanced EGF-induced cell migration and downstream signaling via JNK and Akt, which may explain why ANXA2 knockdown increases lung metastasis formation in mice (31).

The findings of the present study demonstrated that in CaSki cells, ANXA2 acts as an important regulatory factor in EGF-induced EMT. ANXA2 promoted EGF-induced EMT, cell viability and migration activity in CaSki cells in vitro. This suggests that depletion of ANXA2 may structurally and functionally reverse EGF-induced EMT.

\section{Acknowledgements}

We would like to thank Professor Zhaoqi Liu and Dr Changbai Liu at the Institute of Molecular Biology of Three Gorges University (Yichang, China) for their technical advice and assistance. This work was supported by grants from the Projects of Natural Science Foundation of China (grant nos. 81603345 and 81374024) and Projects of Hubei Science foundation (grant no. 2013CFA079). We thank the staff of the Institute of Molecular Biology of Three Gorges University. 


\section{References}

1. Mathur SP, Mathur RS and Young RC: Cervical epidermal growth factor-receptor (EGF-R) and serum insulin-like growth factor II (IGF-II) levels are potential markers for cervical cancer. Am J Reprod Immunol 44: 222-230, 2000.

2. Kim GE, Kim YB, Cho NH, Chung HC, Pyo HR, Lee JD, Park TK, Koom WS, Chun M and Suh CO: Synchronous coexpression of epidermal growth factor receptor and cyclooxygenase- 2 in carcinomas of the uterine cervix: A potential predictor of poor survival. Clin Cancer Res 10: 1366-1374, 2004.

3. Oh MJ, Choi JH, Kim IH, Lee YH, Huh JY, Park YK, Lee KW, Chough SY, Joo KS, Ku BS and Saw HS: Detection of epiderma growth factor receptor in the serum of patients with cervical carcinoma. Clin Cancer Res 6: 4760-4763, 2000.

4. Lee MY, Chou CY, Tang MJ and Shen MR: Epithelial-mesenchymal transition in cervical cancer: Correlation with tumor progression, epidermal growth factor receptor overexpression, and snail up-regulation. Clin Cancer Res 14: 4743-4750, 2008 .

5. Yoshida K, Yoshida S, Choisunirachon N, Saito T, Matsumoto K, Saeki K, Mochizuki M, Nishimura R, Sasaki N and Nakagawa T: The relationship between clinicopathological features and expression of epithelial and mesenchymal markers in spontaneous canine mammary gland tumors. J Vet Med Sci 76 1321-1327, 2014.

6. Gerke V and Moss SE: Annexins: From structure to function. Physiol Rev 82: 331-371, 2002.

7. Flood EC and Hajjar KA: The annexin A2 system and vascular homeostasis. Vascul Pharmacol 54: 59-67, 2011.

8. Brichory FM, Misek DE, Yim AM, Krause MC, Giordano TJ, Beer DG and Hanash SM: An immune response manifested by the common occurrence of annexins I and II autoantibodies and high circulating levels of IL-6 in lung cancer. Proc Natl Acad Sci USA 98: 9824-9829, 2001.

9. Keutzer JC and Hirschhorn RR: The growth-regulated gene 1B6 is identified as the heavy chain of calpactin I. Exp Cell Res 188: $153-159,1990$.

10. Sharma MR, Koltowski L, Ownbey RT, Tuszynski GP and Sharma MC: Angiogenesis-associated protein annexin II in breast cancer: Selective expression in invasive breast cancer and contribution to tumor invasion and progression. Exp Mol Pathol 81: 146-156, 2006.

11. Sharma MR, Rothman V, Tuszynski GP and Sharma MC: Antibody-directed targeting of angiostatin's receptor annexin II inhibits Lewis lung carcinoma tumor growth via blocking of plasminogen activation: Possible biochemical mechanism of angiostatin's action. Exp Mol Pathol 81: 136-145, 2006.

12. Hwang J, Hodis HN, Hsiai TK, Asatryan L and Sevanian A: Role of annexin II in estrogen-induced macrophage matrix metalloproteinase- 9 activity: The modulating effect of statins. Atherosclerosis 189: 76-82, 2006

13. Brownstein C, Deora AB, Jacovina AT, Weintraub R, Gertler M, Khan KM, Falcone DJ and Hajjar KA: Annexin II mediates plasminogen-dependent matrix invasion by human monocytes: Enhanced expression by macrophages. Blood 103: 317-324, 2004

14. Zhao P, Zhang W, Tang J, Ma XK, Dai JY, Li Y, Jiang JL, Zhang SH and Chen ZN: Annexin II promotes invasion and migration of human hepatocellular carcinoma cells in vitro via its interaction with HAb18G/CD147. Cancer Sci 101: 387-395, 2010

15. Sharma M, Ownbey RT and Sharma MC: Breast cancer cell surface annexin II induces cell migration and neoangiogenesis via tPA dependent plasmin generation. Exp Mol Pathol 88 278-286, 2010
16. Ohno Y, Izumi M, Kawamura T, Nishimura T, Mukai K and Tachibana M: Annexin II represents metastatic potential in clear-cell renal cell carcinoma. Br J Cancer 101: 287-294, 2009.

17. Lokman NA, Ween MP, Oehler MK and Ricciardelli C: The role of annexin A2 in tumorigenesis and cancer progression. Cancer Microenviron 4: 199-208, 2011.

18. Serrano MJ, Ortega FG, Alvarez-Cubero MJ, Nadal R, Sanchez-Rovira P, Salido M, Rodríguez M, García-Puche JL, Delgado-Rodriguez M, Solé F, et al: EMT and EGFR in CTCs cytokeratin negative non-metastatic breast cancer. Oncotarget 5: 7486-7497, 2014

19. Bhat FA, Sharmila G, Balakrishnan S, Arunkumar R, Elumalai P, Suganya S, Raja Singh P, Srinivasan N and Arunakaran J: Quercetin reverses EGF-induced epithelial to mesenchymal transition and invasiveness in prostate cancer (PC-3) cell line via EGFR/PI3K/Akt pathway. J Nutr Biochem 25: 1132-1139, 2014.

20. Zheng L, Foley K, Huang L, Leubner A, Mo G, Olino K, Edil BH, Mizuma M, Sharma R, Le DT, et al: Tyrosine 23 phosphorylation-dependent cell-surface localization of annexin A2 is required for invasion and metastases of pancreatic cancer. PLoS One 6: e19390, 2011.

21. Lei C, Wang Y, Huang Y, Yu H, Huang Y, Wu L and Huang L: Up-regulated miR155 reverses the epithelial-mesenchymal transition induced by EGF and increases chemo-sensitivity to cisplatin in human Caski cervical cancer cells. PLoS One 7: e52310, 2012.

22. Huber MA, Kraut N and Beug H: Molecular requirements for epithelial-mesenchymal transition during tumor progression. Curr Opin Cell Biol 17: 548-558, 2005.

23. Mimeault M and Batra SK: Interplay of distinct growth factors during epithelial mesenchymal transition of cancer progenitor cells and molecular targeting as novel cancer therapies. Ann Oncol 18: 1605-1619, 2007.

24. Kersemaekers AM, Fleuren GJ, Kenter GG, Van den Broek LJ, Uljee SM, Hermans J and Van de Vijver MJ: Oncogene alterations in carcinomas of the uterine cervix: Overexpression of the epidermal growth factor receptor is associated with poor prognosis. Clin Cancer Res 5: 577-586, 1999.

25. Noordhuis MG, Eijsink JJ, Ten Hoor KA, Roossink F, Hollema H, Arts HJ, Pras E, Maduro JH, Reyners AK, de Bock GH, et al: Expression of epidermal growth factor receptor (EGFR) and activated EGFR predict poor response to (chemo) radiation and survival in cervical cancer. Clin Cancer Res 15: 7389-7397, 2009.

26. Grewal T and Enrich C: Annexins-modulators of EGF receptor signalling and trafficking. Cell Signal 21: 847-858, 2009.

27. de Graauw M, Cao L, Winkel L, van Miltenburg MH, le Dévedéc SE, Klop M, Yan K, Pont C, Rogkoti VM, Tijsma A, et al: Annexin A2 depletion delays EGFR endocytic trafficking via cofilin activation and enhances EGFR signaling and metastasis formation. Oncogene 33: 2610-2619, 2014.

28. Cha YH, Kim NH, Park C, Lee I, Kim HS and Yook JI: MiRNA-34 intrinsically links p53 tumor suppressor and Wnt signaling. Cell Cycle 11: 1273-1281, 2012.

29. Zhou S, Yi T, Liu R, Bian C, Qi X, He X, Wang K, Li J, Zhao X, Huang $\mathrm{C}$ and Wei Y: Proteomics identification of annexin A2 as a key mediator in the metastasis and proangiogenesis of endometrial cells in human adenomyosis. Mol Cell Proteomics 11: M112, 2012.

30. Yim EK, Tong SY,Ho EM,Bae JH,Um SJ and Park JS: Anticancer effects on TACC 3 by treatment of paclitaxel in HPV-18 positive cervical carcinoma cells. Oncol Rep 21: 549-557, 2009.

31. Andey T, Marepally S, Patel A, Jackson T, Sarkar S, O'Connell M, Reddy RC, Chellappan S, Singh P and Singh M: Cationic lipid guided short-hairpin RNA interference of annexin A2 attenuates tumor growth and metastasis in a mouse lung cancer stem cell model. J Control Release 184: 67-78, 2014. 